\title{
Acétylcholine et plasticité neuronale du cortex somatosensoriel
}

Le prosencéphale sous-frontal contient des neurones cholinergiques qui projettent dans toutes les régions corticales. L'acétylcholine ainsi libérée prolongerait la dépolarisation de neurones corticaux excités, augmentant, de ce fait, leur réponse. Ce système permettrait, par conséquent, de moduler la réponse à des stimulations somatosensorielles et pourrait ainsi être impliqué dans des processus d'apprentissage et de mémorisation.

\section{Robert W. Dykes}

\section{ADRESSE}

R.W. Dykes : professeur titulaire. Centre de recherches en sciences neurologiques, faculté de médecine, département de physiologie,

université de Montréal, Montréal, Québec.

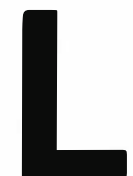

a fiabilité opérationnelle du système nerveux est une condition essentielle à la survie de l'être humain. Marcher ou courir avec l'assurance que le prochain pas sera identique aux précédents repose sur le caractère reproductible de la coordination de nos mouvements et de notre perception sensori-motrice. Néanmoins, notre vie dépend également de notre capacité d'apprentissage et de mémorisation. D'un côté, notre système nerveux est donc caractérisé par sa rigidité tant sur le plan fonctionnel que sur celui de ses connexions, comparable à celle d'un ordinateur; il doit effectuer et répéter les tâches avec régularité et précision. D'un autre côté, toutefois, son activité implique des changements importants du fonctionnement neuronal, pendant des périodes prolongées. Un des mystères du cerveau est cette intégration dans une seule et unique structure de deux attributs diamétralement opposés.
Nous prenons ici comme base que la reproductibilité des réponses neuronales est liée à leur morphologie et à leurs connexions, établies au cours du développement. Le problème posé est donc de savoir comment, chez l'adulte, les fonctions neuronales peuvent être changées.

Il existe au moins deux mécanismes possibles à l'origine de telles modifications de l'activité neuronale : (1) l'établissement de nouvelles connexions ou (2) un changement de la puissance de connexions existantes. Au stade périnatal, l'activité neuronale change avec l'établissement de nouvelles connexions. Chez les mammifères adultes, en revanche, une telle modification découle le plus souvent de changements dans l'efficacité de connexions déjà en place $[1,2]$. Ces changements, que nous appellerons plasticité neuronale, nécessitent que l'animal soit éveillé, ou du moins que le système d'éveil soit activé. Singer et ses collaborateurs [3] ont vérifié cette hypothèse chez le chaton 
où ils n'ont pu produire une plasticité neuronale dans le cortex visuel pendant le sommeil ou sous anesthésie, ce qui les a conduits à la conclusion que l'état de veille était une condition " permissive " à la plasticité neuronale. Ces données nous permettent d'identifier la région du prosencéphale sous-frontal (PSF) comme une structure fondamentale pour l'existence des processus de plasticité neuronale. Cette région du cerveau, méconnue, contient les neurones à l'origine de plusieurs systèmes neuromodulateurs importants. L'un de ces systèmes, dont les neurones contiennent de l'acétylcholine ( $\mathrm{ACh}$ ), est impliqué dans les mécanismes d'éveil et de plasticité neuronale du cortex. Chez la plupart des mammifères, les afférences provenant de la région PSF sont la seule source d'acétylcholine du cortex cérébral. Chaque axone du PSF n'innerve qu'une région restreinte du cortex, mais l'ensemble des projections de ces neurones pénètre toutes les régions du cortex et y apporte l'acétylcholine (figure 1).

\section{Les effets \\ de l'acétylcholine dans le cortex cérébral}

Depuis des décennies, il est établi que la libération d'ACh est beaucoup plus élevée pendant la veille que pendant le sommeil, mais les effets spécifiques de l'ACh sur les neurones corticaux restent méconnus.

Krnjevic et Phillis $[4,5,6]$ ont entrepris cette étude lors d'une expérience remarquable durant laquelle ils ont enregistré l'activité des neurones avant, pendant et après l'application d'ACh par iontophorèse au travers d'une micropipette accolée à la pipette d'enregistrement. Les observations recueillies lors de ces expériences chez des chats constituent la base de nos connaissances actuelles des effets de l'ACh sur les neurones corticaux. Ces auteurs ont observé que seuls 20 à $30 \%$ des neurones étaient affectés par cette application et que, fait surprenant, l'administration de larges quantités d'ACh n'avait aucun effet sur la plupart des cellules.

La réponse des neurones sensibles à l'ACh n'était pas, de plus, impres$m / s n^{\circ} 9$, vol. 6 , novembre 90

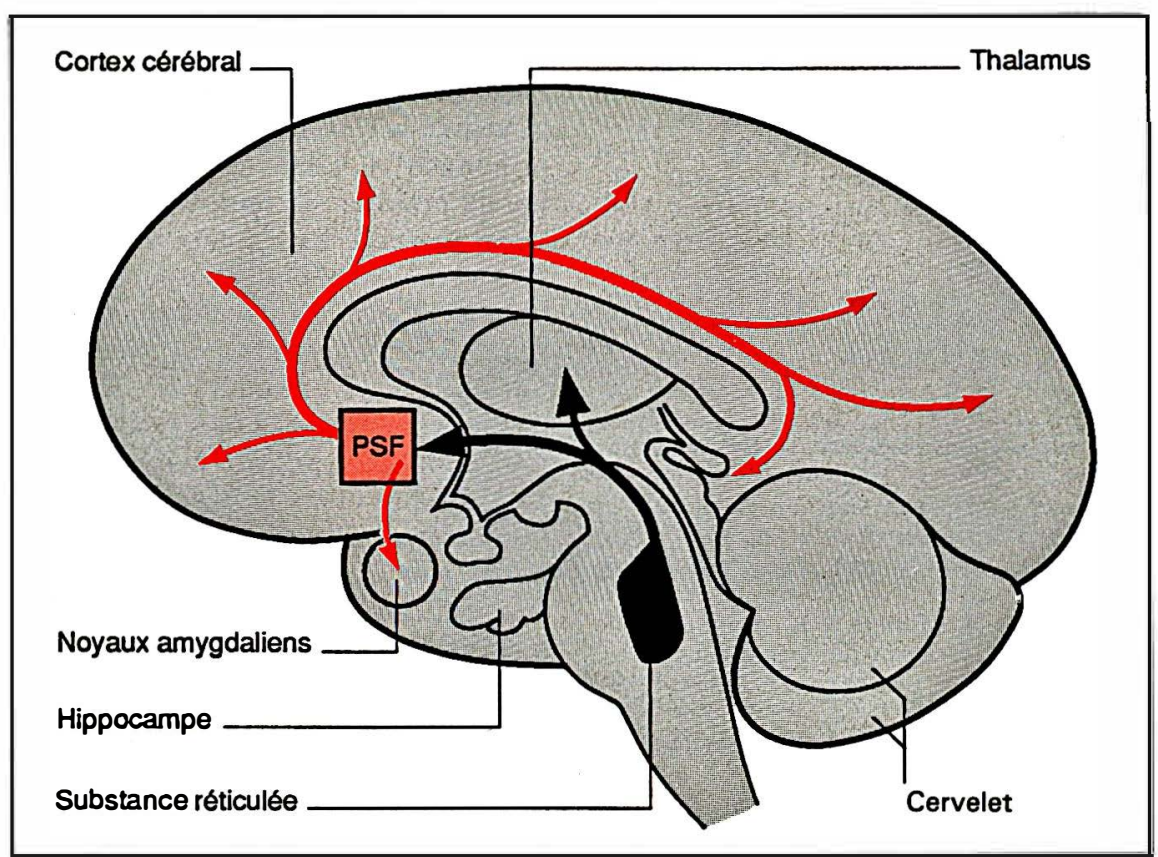

Figure 1. Le système cholinergique. "I n'y a aucun neurone cholinergique dans le cortex, chez la plupart des mammifères. La seule source d'acétylcholine (ACh) provient d'une collection de neurones localisée dans une partie du cerveau, identifiée comme prosencéphale sous-frontal (PSF). Les axones de ces cellules s'étendent dans toutes les régions du cortex, chacune en desservant une partie restreinte. Une des voies principales de l'activation du PSF est la formation réticulée activatrice du tronc cérébral. Cette structure, mise en marche par divers stimuli provenant de l'environnement, amène le PSF à libérer I'ACh de ses terminaisons sur de vastes étendues du cortex.

sionnante ; selon l'intensité du courant d'éjection, le neurone affecté ne commence à répondre, par ailleurs, qu'après un certain délai dont la durée peut aller jusqu'à 20 secondes [7].

On sait à présent que ces effets de l'ACh au niveau cortical se manifestent à la suite de sa liaison avec des récepteurs membranaires de type muscarinique [8]. Il n'existe dans le cortex cérébral qu'une faible proportion de récepteurs de type nicotinique qui, pour leur part, produisent des effets immédiats et de courte durée, fort probablement de la même façon qu'au niveau de la jonction neuromusculaire. On pense aujourd'hui qu'il existe encore un troisième type de récepteurs situé sur les interneurones corticaux inhibiteurs [9]. D'une façon générale, il semble que l'ACh ait un effet excitateur et que l'on ne rencontre d'effets inhibi- teurs que lorsque ceux-ci sont indirectement produits par la stimulation d'interneurones inhibiteurs au voisinage du neurone enregistré [10].

On a pu explorer plus avant les mécanismes neuronaux intrinsèques à l'aide d'enregistrements intracellulaires. On a ainsi démontré que l'administration d'ACh au voisinage du neurone produisait une baisse de la conductance transmembranaire au potassium $[8,11]$. La diminution de la conductance du potassium diminuerait la vitesse du retour de la cellule à son potentiel de repos et augmenterait, par voie de conséquence, le nombre de potentiels d'action émis durant la repolarisation [12].

En l'absence d'une dépolarisation dans la plupart des neurones corticaux, les effets de l'ACh sur les neurones dépendent de leur état au moment de l'application. Même si les changements membranaires ont peu 


\section{RÉFÉRENCES}

1. Merzenich MM, Recanzone G, Jenkins WM, Allard TT, Nudo RJ. Cortical representational plasticity. In : Rakic $P$, Singer W, eds. Neurobiology of Neocortex. Dahlem Konferenzen. London : John Wiley and sons, 1988 : 41-67

2. Snow $P J$, Nudo RJ, Rivers $W$, Jenkens WM, Merzenich MM. Somatotopically inappropriate projections from thalamocortical neurons to the SI cortex of the cat demonstrated by the use of intracortical microstimulation. Somalosensory Res 1988 ; 5 : 349-72.

3. Singer $W$. Neuronal mechanisms of experience-dependent self-organization of the mammalian visual cortex. Acta Morphol Hung 1983 ; 31 : 235-60.

4. Krnjevic K, Phillis JW. Iontophoretic studies of neurones in the mammalian cerebral cortex. I Physiol (Lond) 1963 ; 165 : 274-304.

5. Krnjevic K, Phillis JW. Acetylcholinesensitive cells in the cerebral cortex. $J$ Physiol (Lond) 1963 ; 165 : 296-327

6. Krnjevic K, Phillis JW. Pharmacological properties of acetylcholine-sensitive cells in cerebral cortex. $J$ Physiol (Lond) 1963 ; $166: 328-50$

7. Lamour Y, Dutar P, Jobert A. Excitatory effect of acetylcholine on different types of neurons in the first somatosensory neocortex of the rat : laminar distribution and pharmacological characteristics. $J$ Neurosci 1982 ; 7 : 1483-94.

8. McCormick DA. Cholinergic and noradrenergic modulation of thalamic processing. Trends Neurosci $1989 ; 12$ : 215-21.

9. McCormick DA, Prince DA. Two types of muscarinic response to acetylcholine in mammalian cortical neurons. Proc Natl Acad Sci USA 1985; 82 : 6344-8.

10. Muller CM, Singer W. Acetylcholineinduced inhibition in the cat visual cortex is mediated by a GABAergic mechanism. Brain Res 1989 ; 487 : 335-42.

11. Krnjevic K, Pumain R, Renaud L. The mechanism of excitation by acetylcholine in the cerebral cortex. J Physiol (Lond) $1971 ; 215$ : 247-68.

12. Woody CD, Gruen E. Acetylcholine reduces the outward currents measured in vivo with single electrode voltage clamp techniques in neurons of the motor cortex of cats. Brain Res 1987 ; 424 : 193-8.

13. Nichols RA. The coupling of neurotransmitter receptors to ion channels in the brain. Science 1988 ; 241 : 545-51.

14. Bear MF, Kleinschmidt A, Gu Q, Singer W. Disruption of experience-dependent synaptic modifications in striate cortex by infusion of NMDA receptor antagonist. $J$ Neurosci 1990 ; 10 : 909-25.

15. Fisher RS, Bushwald NA, Hull CD, Levine MS. GABAergic basal forebrain neurons project to the neocortex : the localization of glutamic acid decarboxylase and choline acetyltransferase in feline corticopetal neurons. I Comp Neurol 1988 ; 272 : 489-502.

16. Richardson RT, Delong MR. Nucleus basalis of Meynert neuronal activity during a delayed response task in monkey. Brain d'effet sur une cellule inactive, la liaison de l'ACh intervient, aussitôt son entrée en activité, pour permettre à la cellule d'être dépolarisée de façon prolongée. Chacun de ces changements prolonge le temps d'ouverture des canaux associés aux récepteurs NMDA (N-méthyl-D-aspartate) et favorise l'entrée du calcium dans la cellule. Les réactions enzymatiques intracellulaires induites par ces mécanismes sont responsables des changements de la fonction neuronale [8, $13,14]$. Les effets de l'ACh se manifestent sur des neurones activés à partir d'une autre voie, puisque les changements membranaires sont consécutifs à l'entrée de calcium dans la cellule. Cela revient à dire que le neurone doit être préalablement dépolarisé d'une façon ou d'une autre pour que l'ACh ait un effet sur lui. Ceci constitue une idée maîtresse dans la compréhension du rôle de l'ACh dans le cortex.

\section{Le fonctionnement du PSF durant des tests comportementaux}

Mis à part l'éveil, dans quelles situations observe-t-on des changements d'activité des neurones du PSF ? Il y a plusieurs difficultés à surmonter pour obtenir ces informations. Premièrement, on doit introduire des électrodes jusqu'au PSF pour enregistrer l'activité neuronale ; celle-ci doit être recueillie au moment où l'animal est conscient et travaille en vue d'une récompense. Deuxièmement, il faut prouver que les neurones enregistrés projettent bien vers le cortex en activant de façon antidromique les neurones du PSF. Troisièmement, il faudrait également parvenir à démontrer que ces neurones libèrent bien de l'ACh, ce qui est difficile. Les neurones cholinergiques sont en effet dispersés dans le PSF, où ils coexistent avec des neurones non cholinergiques. Des neurones du PSF contenant du GABA, par exemple, possèdent également des projections corticales [15]. Enfin, il est nécessaire de contrôler le comportement de l'animal de façon répétée : si l'animal ne parvient pas à accomplir la tâche qu'on lui assigne, il est impossible de déterminer s'il existe une relation entre celle-ci et l'activité neuronale.
Tout cela permet de comprendre pourquoi il n'existe pas, à l'heure actuelle, un vaste éventail de travaux portant sur les neurones du PSF. Certaines données intéressantes ont cependant été recueillies. Richardson et DeLong [16] et Richardson et al. [14] ont ainsi enregistré l'activité des neurones PSF chez un singe entraîné à déplacer un levier pour atteindre une cible puis à attendre plusieurs secondes avant de recevoir sa récompense. L'activité de nombreux neurones (plus de $50 \%$ ) a changé pendant la période où le singe attendait la récompense et après la remise de celle-ci, suggérant aux auteurs une participation de cette population neuronale dans des mécanismes de " renforcement positif " et d'apprentissage. Poursuivant cette étude, ils ont observé une modification constante de l'activité dans plusieurs types de situations expérimentales, dans les secondes précédant immédiatement la récompense. Cela les a amenés à conclure que les neurones du PSF étaient particulièrement sensibles à des événements s'accompagnant d'une récompense.

Très récemment, Wilson et Rolls [18] ont repris cette étude chez des singes entraînés à répondre à deux signaux différents, l'un annonçant la remise d'une récompense et l'autre l'application d'un stimulus aversif. Sur 2000 neurones du PSF enregistrés, 120 ont montré un changement d'activité lié à la remise de la récompense. Ces neurones semblent donc capables de répondre à un signal lorsque celui-ci a été associé à un événement lié à une récompense. Ces changements d'activité ont été observés après que le signal a été présenté une seule fois parmi 30 essais, ce qui suggère que l'activité neuronale est associée à un phénomène d'apprentissage et de mémorisation. Ceci a amené les auteurs à conclure que les neurones du PSF pourraient jouer un rôle facilitateur dans l'établissement de la mémoire dans les diverses zones corticales, en agissant par le biais de mécanismes liés à l'attention.

\section{L'acétylcholine et la plasticité neuronale}

Jusqu'à très récemment, il n'avait 
cependant pas été possible d'établir un lien entre une libération d'ACh et une amélioration des performances au niveau cortical chez l'animal conscient, alors même que l'on connaissait des corrélations entre libération d'ACh et performance d'une part, activité neuronale et apprentissage d'autre part. Woody et al. [19] ont, les premiers, démontré indirectement l'existence d'une telle corrélation en utilisant un protocole classique de conditionnement. Dans ce conditionnement, un jet d'air est utilisé pour provoquer un battement de paupières chez des chats alors qu'on enregistre, par voie intracellulaire, l'activité des neurones du cortex moteur. L'activité de ces neurones change pendant la période d'apprentissage et peut rester ensuite modifiée pendant plusieurs jours sauf lorsque l'on bloque les sites récepteurs muscariniques de l'ACh. Au cours de ces études, les auteurs ont observé que l'augmentation de l'activité neuronale associée au réflexe conditionné a lieu en même temps qu'une libération d'ACh s'accompagnant d'un accroissement de la résistance membranaire. Dans une seconde série d'expériences, le même effet fut observé lors de l'administration intracellulaire d'ACh et d'un courant dépolarisant. Dès lors, on a établi un lien entre apprentissage et $\mathrm{ACh}$ par ce biais du conditionnement.

Les divers travaux résumés ci-dessus ont démontré que la présence d'ACh était liée à une potentialisation de l'activité neuronale et à une variation de cette activité en fonction de l'état de vigilance. Toutes ces informations ne nous renseignent pas, toutefois, sur la façon dont l'ACh permet la plasticité.

Pour vérifier cette association entre présence d'ACh et plasticité neuronale, Metherate et al. [20, 21] ont utilisé les techniques d'iontophorèse, comme Kmjevic et ses collaborateurs, et ont appliqué de l'ACh près de neurones du cortex somesthésique dépolarisés, par application de glutamate. Ils ont ainsi montré [21] qu'une telle application augmente l'excitabilité du neurone non seulement pendant la période d'administration, mais encore pour une longue période de temps (jusqu'à une heure), même si le traitement $\mathrm{ACh}$ $\mathrm{m} / \mathrm{s} n^{\circ} 9$, vol. 6 , novembre 90 n'est que de très courte durée (une minute ou moins). L'effet de l'ACh n'est que permissif puisqu'une dépolarisation est essentielle à l'obtention d'une action à long terme, l'application d'ACh entre des cycles de dépolarisation au glutamate ou en présence d'un bloquant muscarinique (atropine) ne produisant aucun effet. Des effets comparables ont été observés dans le cortex somesthésique du rat par Lamour et al. [22]. On peut donc conclure que les deux conditions nécessaires au prolongement de l'excitabilité neuronale sont, d'une part, un état de dépolarisation et, d'autre part, une liaison de l'ACh aux récepteurs muscariniques.

Dans le cortex somesthésique, les neurones sont activés par stimulation

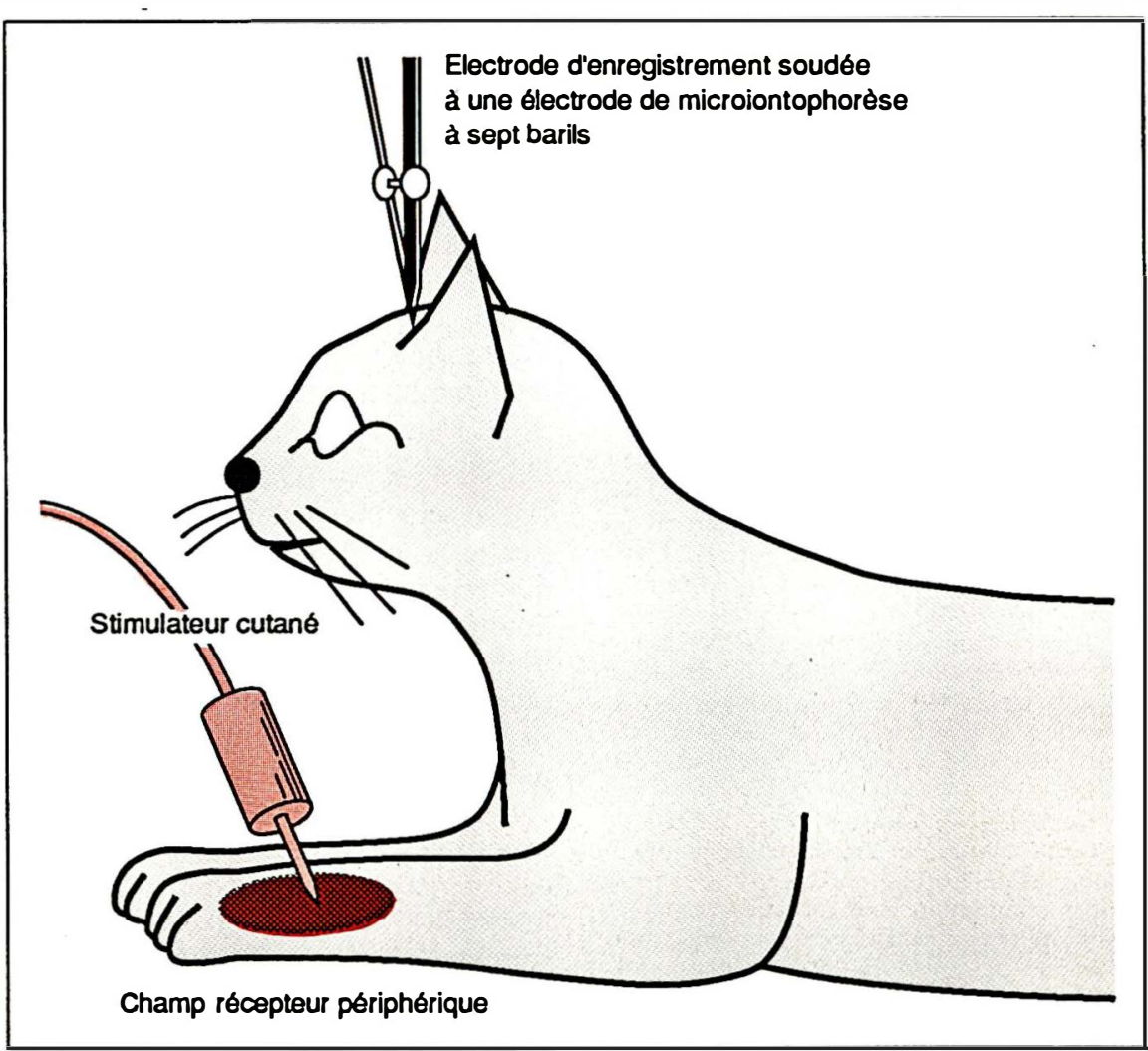

Figure 2. La technique d'iontophorèse consiste à libérer de petites quantités de substances proportionnellement à la quantité d'un courant passé par le fût d'une pipette multicanal. Pendant l'administration d'une substance, un courant de retenue est appliqué aux autres fûts pour prévenir la fuite des autres substances. A l'aide d'une seconde pipette, placée en parallele de l'électrode de microiontophorèse, on peut observer l'activité neuronale dans le voisinage. Par des ajustements délicats de cet assemblage, on peut enregistrer un neurone isolé et étudier son comportement en présence de substances pharmacologiques. En même temps, à l'aide d'un stimulateur cutané, il est possible d'administrer des stimulations mécaniques calibrées et d'observer les réponses neuronales et leurs changements avec ou sans administration d'ACh. 
17. Richardson RT, Mitchel SJ, Baker FH, Delong MR. Responses of nucleus basalis of Meynert neurons in behaving monkeys. In : Woody CD, Alkon DL, McGaugh JL, eds. Cellular Mechanisms of Conditioning and Behavioral Plasticity. New York : Plenum Publishing, 1988: 161-73.

18. Wilson FAW, Rolls ET. Learning and memory are reflected in the responses of reinforcement related neurons in the primate basal forebrain. J Neurosci 1990; 10 : 1254-67.

19. Woody CD, Swartz BE, Gruen E. Effects of acetylcholine and cyclic GMP on input resistance of cortical neurons in awake cats. Brain Res 1978 ; 158 : 373-95.

20. Metherate R, Tremblay N, Dykes RW. The effects of acetylcholine on response properties of cat somatosensory cortical neurons. J Neurophysiol 1988 ; 59 : 1231-52.

21. Metherate R, Tremblay N, Dykes RW. Transient and prolonged effects of acetylcholine on responsiveness of cat somatosensory cortical neurons. J Neurophysiol 1988 ; 59 : 1253-76.

22. Lamour Y, Dutar P, Jobert A, Dykes RW. An iontophoretic study of single somatosensory neurons in rat granular cortex serving the limbs : a laminar analysis of glutamate and acetylcholine effects on receptive field properties. J Neurophysiol 1988 ; 60 : 725-50.

23. Tremblay N, Warren R, Dykes RW. Electrophysiological studies of acetylcholine and the role of the basal forebrain in the somatosensory cortex of the cat. (I) Cortical neurons excited by glutamate. $J$ Neurophysiol 1990 ; 64 : 1199-211.

24. Tremblay N, Warren R, Dykes RW. Electrophysiological studies of acetylcholine and the role of the basal forebrain in the somatosensory cortex of the cat. II. Cortical neurons excited by somatic stimuli. $J$ Neurophysiol 1990 ; 64 : 1212-22.

25. Rasmusson DD, Dykes R.W. Longterm enhancement of evoked potentials in cat somatosensory cortex produced by coactivation of the basal forebrain and cutaneous receptors. Exp Brain Res 1988 ; 70 : 276-86.

26. Greuel JM, Luhman HJ, Singer W. Persistent changes of single-cell responses in kitten striate cortex produced by pairing sensory stimulation with iontophoretic application of neurotransmitters and neuromodulators. In : Woody CD, Alkon DL, McGaugh JL, eds. Cellular Mechanisms of Conditioning and Behavioral Plasticity. New York: Plenum Press, 1988 : 425-36.

27. Dykes RW, Tremblay N, Warren P. Neuromodulatory substances, somatosensory cortical neuronal responsiveness and longterm changes in neuronal excitability. In : Franzen $\mathrm{O}$, Westman J, eds. Information processing in the Somatosensory System : Proceedings of an International Symposium Held at the Wenner-Gren Center Stockholm, July 3-5, 1989. 1990.

28. Lynch G, Muller D, Seubert P, Larson $\mathrm{J}$. Long-term potentiation : persisting problems and recent results. Brain Res Bull $1988 ; 21: 363-72$
On peut cependant critiquer dans ces expériences, l'utilisation de paramètres de stimulation non physiologiques et de concentrations d'ACh plus élevées que normalement dans le cerveau. Afin de nous rapprocher de quantités d'ACh libérées plus physiologiques, nous avons poursuivi ces études [23, 24] en insérant des électrodes de stimulation dans le PSF pour stimuler les neurones cholinergiques. L'étude a consisté à activer les neurones du cortex somesthésique par stimulation cutanée chez le chat, sous anesthésie, alors que l'on stimulait électriquement, ou non, les neurones du PSF (figure 2). L'enregistrement des potentiels évoqués dans le cortex somesthésique a montré que la réponse était, de façon stable, augmentée de $98 \%$ pendant toute la durée de l'expérience, soit environ 4 à 5 heures, et ce même après une seule série de stimulations électriques du PSF [25]. La libération d'ACh par stimulation électrique du PSF, si elle a lieu au cours de la dépolarisation d'un neurone, provoque donc un accroissement de l'excitabilité neuronale. Cet effet, qui est de très longue durée, pourrait servir d'appui à une théorie générale sur les modifications du système nerveux regroupées sous les termes de plasticité neuronale.

Il est cependant difficile de démontrer directement que les modifications des réponses observées après stimulation électrique du PSF sont dues à la libération d'ACh [25]. De plus, un potentiel évoqué cortical n'est qu'une mesure indirecte des fonctions neuronales. Tremblay et al. [23, 24] ont comblé les lacunes des expériences rapportées ci-dessus en réalisant des expériences d'enregistrement unicellulaire à l'aide d'électrodes d'enregistrement couplées à une électrode d'iontophorèse à fûts multiples. Ils ont quantifié les réponses neuronales enregistrées pendant une stimulation systématique et constante du champ récepteur cutané en présence, ou non, d'une ou de plusieurs séries de stimulations électriques du PSF. De telles stimulations provoquent un accroissement des réponses de 30 à $450 \%$ (moyenne : $120 \%$ ), qui est bloqué par l'administration d'atropine juste avant la stimulation (figure 3). On peut donc conclure que l'ACh a un effet sur la réponse neuronale, à condition que son application soit couplée à une dépolarisation membranaire. Greuel et al. [26] ont suggéré qu'un mécanisme semblable intervenait dans le cortex visuel en montrant que les modifications du fonctionnement neuronal qui permettent l'établissement des caractéristiques de dominance oculaire ne se produisent pas en l'absence de libération d'ACh et/ou de noradrénaline.

\section{La théorie, ses forces et ses faiblesses}

Sur la base de ces observations, il est possible de formuler l'hypothèse selon laquelle le cortex somesthésique luimême serait responsable de l'analyse systématique et régulière des signaux induits par stimulation sensorielle. A paramètres constants de stimulation sensorielle, la libération d'ACh couplée à l'activité neuronale provoque l'accroissement de la réponse neuronale. Le cortex a ainsi la capacité d'améliorer sa réponse lorsque les signaux qui lui parviennent sont de faible intensité.

Un même type de mécanisme peut expliquer la cascade de changements qui surviennent dans la carte somesthésique corticale à la suite d'une désafférentation. La modification de l'activité d'un neurone par la perte de son champ récepteur peut, en effet, influencer les neurones avoisinants et engendrer une série de modifications kaléiodoscopiques dans la carte somesthésique corticale, jusqu'à obtention d'un nouvel état stable [27]. Une telle hypothèse soulève cependant plusieurs problèmes, notamment parce que l'on ne connaît pas la durée réelle de cet effet et que l'on n'est pas en mesure de savoir si le changement opéré est permanent ou transitoire. Il est toutefois possible de dire que la durée de cet effet est suffisante pour permettre une comparaison avec les phénomènes de potentialisation à long terme dans l'hippocampe et le relier, donc, à des mécanismes de mémorisation [28]. Si nous sommes en présence de phénomènes liés à la mémoire, leurs mécanismes d'action imposent la suppression d'activités neuronales indésirables, telles celles liées aux réponses incorrectes lors de l'apprentissage 


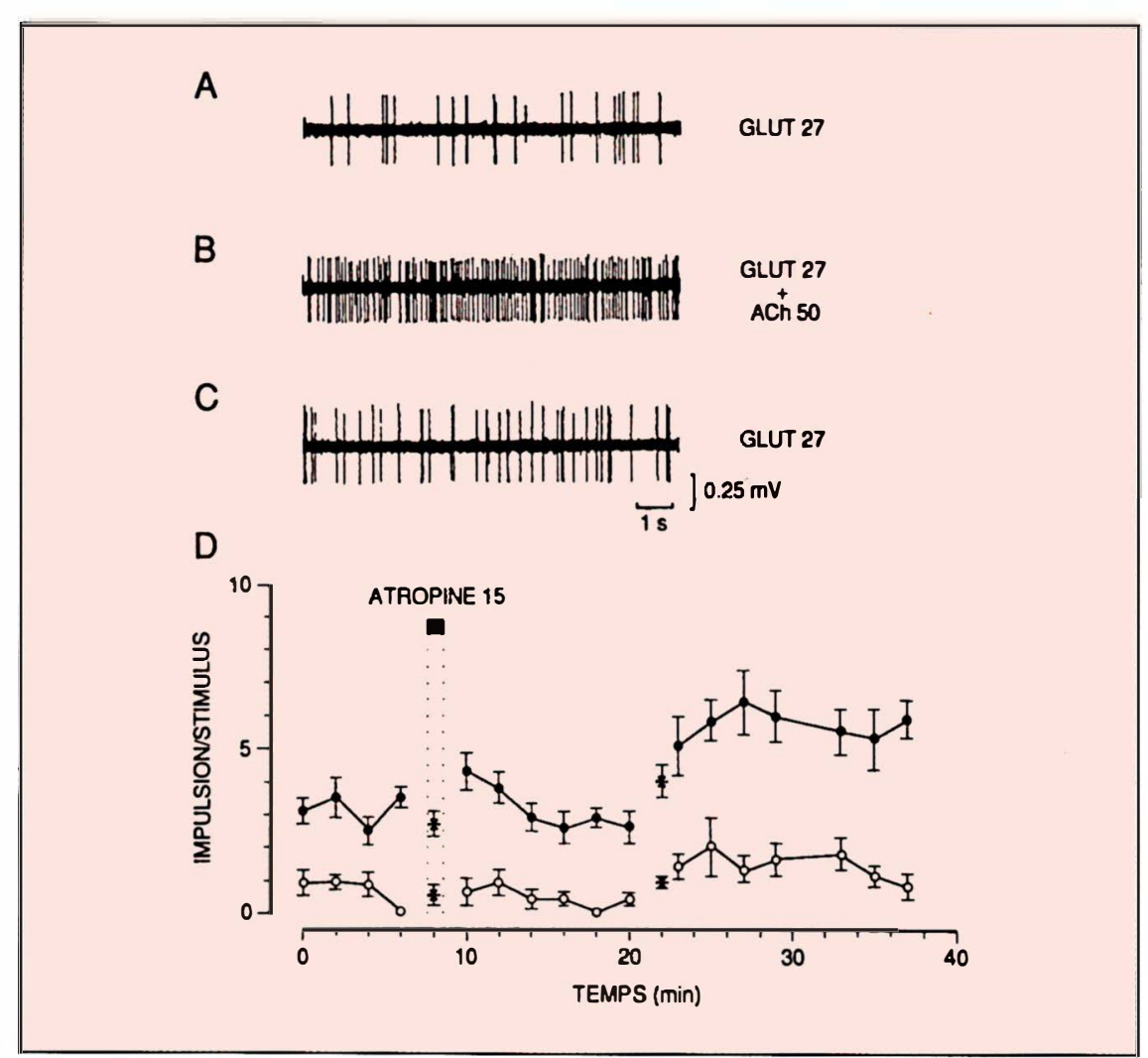

Figure 3. Tracés illustrant l'effet facilitateur de I'ACh sur une cellule répondant à l'application de glutamate. Chacun des tracés illustre la phase initiale (10 sec.) d'une application d'une durée totale de 1 minute. A : réponse contrôle à l'application d'un courant de $27 n A$ de glutamate. B : l'application $d^{\prime} u n$ courant de $50 n A d^{\prime} A C h$ augmente la réponse au glutamate. $C$ : retour à la valeur contrôle 2 min après l'interruption de l'application de l'ACh. Cet effet à court terme est semblable à celui de l'état de veille. $D$ : l'effet facilitateur induit par la stimulation du PSF (*) sur la réponse évoquée par stimulation cutanée (chaque point représente la moyenne de 10 stimulations) est bloqué en présence d'atropine. En revanche, l'absence d'atropine pendant la seconde stimulation du PSF simultanément avec celle du champ récepteur provoque une augmentation significative et à long terme de la réponse neuronale aux stimulations subséquentes.

d'une tâche. Le mécanisme décrit cidessus ne fournit pas de données sur les mécanismes aboutissant à une diminution d'excitabilité de neurones [14]. Une autre question qui reste en suspens est celle des changements d'état de vigilance associés à une libération d'ACh. On peut, en effet, se poser la question de savoir pourquoi les réponses neuronales ne sont pas en permanence augmentées au cours de la veille, alors que les taux d'ACh libérée sont nettement accrus. En l'absence d'une solution satisfaisante à ce problème, on ne peut que noter que les concentrations d'ACh sont plus fortes encore pendant les périodes de plasticité neuronale que pendant la veille.

Il est évident que beaucoup de che$\mathrm{m} / \mathrm{s} n^{\circ} 9$, vol. 6 , novembre 90 die d'Alzheimer. Il est probable, en effet, que la maladie d'Alzheimer est plus complexe qu'une dégénérescence graduelle et progressive des neurones cholinergiques. Ces recherches ont d'ores et déjà montré que la perte des neurones cholinergiques peut engendrer des troubles sévères dans l'activité des neurones corticaux, en particulier dans leur capacité à répondre, par la plasticité, à l'apparition de nouvelles exigences

\section{Summary}

Acetylcholine and neural plasticity of the somatosensory cortex

The basal forebrain contains cholinergic neurons which are the only source of acetylcholine released in the cortex of most mammals. The axons of these cells project throughout cortex and are found in all cortical layers. The released acetylcholine prolongs the depolarization of cortical neurons but of ten has little apparent effect unless another source of excitation causes a depolarization to occur, at which time acetylcholine enhances the effect of this other signal. This arrangement allows the basal forebrain to modulate the ongoing neuronal activity in somatosensory cortex and to be an important aspect of consciousness and directed attention. The work performed in the author's laboratory demonstrates that when acetylcholine is presented during periods of depolarization, the excitability of the depolarized somatosensory cortical neuron is enhanced for hours at a time. This observation suggests that the release of acetylcholine is involved in processes of learning and memory.

\section{Remerciements}

Sans l'appui et la générosité de mes collègues, ce premier article n'aurait jamais pu paraître en français, langue que je suis encore loin de maîtriser. Je désire exprimer ma reconnaissance à Madame Monique Turcotte, à mon étudiant Serge Leclerc et à mes collègues Marc Peschanski et Laurent Descarries.

\section{TIRÉS A PART}

R.W. Dykes. 